\title{
A Study on Bacterial Profile and Antibiotic Susceptibility Pattern in Complicated Urinary Tract Infection in a Tertiary Care Centre
}

\author{
B. Natesan* and R. Manjula \\ Institute of Microbiology, Madras Medical College and RGGGH, Chennai, Tamil Nadu, India \\ *Corresponding author
}

\begin{tabular}{|c|c|}
\hline & A B S T R A C T \\
\hline & \multirow{6}{*}{$\begin{array}{l}\text { Complicated urinary tract infections (cuti) refer to those infections that occur in the } \\
\text { presence of anatomic or functional abnormalities in the kidney. Urine from } 120 \\
\text { complicated UTI patients was collected and subjected to gram staining, culture and } \\
\text { sensitivity by routine methods and ESBL resistance detected by screening and phenotypic } \\
\text { methods. Males contributed to maximum number of isolates with } 67.5 \% \text {. Maximum } \\
\text { number of isolates was obtained in the } 51-60 \text { yrs age group. The gram negative bacill } \\
\text { constituted } 90.83 \% \text { of isolates and gram positive cocci constituted } 9.17 \% \text { of isolates. E } \\
\text { coli was the commonest isolate with } 40.83 \% \text { and Klebsiella spp with } 33.33 \% \text {. The othe } \\
\text { isolates were Pseudomonas spp, Acinetobacter spp, S. aureus, Citrobacter spp and Proteu } \\
\text { vulgaris. In GNB isolates, Amikacin, Nitrofurantoin and Imipenem had higher sensitivity } \\
\text { The gram positive cocci isolated were Enterococci spp and S. aureus which were } 7 \text { and } 4 \\
\text { isolates respectively. The ESBL production in E. coli was higher than Klebsiella spp. The } \\
\text { high rate of resistance demands an effective drug control policy to be formulated by the } \\
\text { knowledge obtained in this study for the treatment of these patients. }\end{array}$} \\
\hline & \\
\hline $\begin{array}{l}\text { Comp } \\
\text { Imipe } \\
\text { Drug } \\
\text { E. col }\end{array}$ & \\
\hline Article & \\
\hline $\begin{array}{l}\text { Accepte } \\
21 \text { Sept } \\
\text { Availab } \\
10 \text { Octo }\end{array}$ & \\
\hline & \\
\hline
\end{tabular}

\section{Introduction}

Complicated urinary tract infections (c UTI) refer to those infections that occur in the presence of anatomic or functional abnormalities in the kidney, bladder or collecting systems. A complicated infection is associated with factors that increase the chance of acquiring bacteria and decrease the efficacy of therapy. The host is compromised and / or the bacteria have increased virulence [or] antimicrobial resistance.

Upto $40 \%$ to $50 \%$ of the female population will experience asymptomatic UTI at some time during their life and about $3 \%$ of all women in the United States visit a physician each year for a UTI (Foxman et al., 2002).
Men with bacteriuria frequently have anatomic abnormalities of the urinary tract. The uropathogens causing cUTI and their antibiotic sensitivity patterns varies considerably not only from that in acute uncomplicated UTI but also with time. The ascending route by way of the urethra is the most usual way by which micro-organisms enter the urinary tract.

$E$ coli was the most common organism causing c UTI in a study done by Peterson et al., ( 2007) in the United states. Strains of $E$. coli that infect patients with complicating factors [or] invasive procedures on the urinary tract, may lack specialized virulence factors. 
Antimicrobial resistance rates of these strains vary and are highest among nosocomial strains under intense selection pressure in the hospital environment. ESBL producing organism poses a major problem for clinical therapeutics. The incidence of ESBL producing strains among clinical isolates have been on steady increase posing a problem in therapeutic options.

The knowledge of drug resistance pattern in a geographical area and the formulation of an appropriate hospital antibiotic policy will go a long way in the control of these infections. Therefore it is necessary to know the antibiotic susceptibility pattern of pathogens to select the correct antibiotic(s) for proper treatment of cUTI.

Knowledge of the microbial organisms and antibiotic susceptibility pattern of c UTI is essential for defining empirical treatment.

The main aim of this study to determine the Bacteriological profile in patients with Complicated Urinary Tract Infection and determine the Antibiotic susceptibility pattern using Kirby Bauer Disc diffusion method. Also identify the prevalence of ESBL in $E$. coli and Klebsiella spp by screening and phenotypic confirmatory methods. Finally, analyse the drug resistant pattern in uropathogens in cUTI.

\section{Materials and Methods}

\section{Sample collection}

Urine collection from women by clean catch midstream technique requires personal supervision for best results. The periurethral area and perineum are first cleansed with two or three gauze pads saturated with soapy water, using a forward - to - back motion, followed by a rinse with sterile saline or water.
The labia should be held apart during voiding, and the first few milliliters of urine passed into a bedpan or toilet bowl to flush out bacteria from the urethra. The midstream portion of urine is then collected in a sterile, wide-mouthed container that can be covered with a tightly fitted lid. The soapy water preparation is usually not required for men; rather, simple cleansing of the urethral meatus immediately after retraction of prepuce before voiding and then collection of the midstream sample is usually sufficient.

Specimens should be processed within 2 hours after collection to achieve accurate colony counts.

\section{Urine culturing and bacterial identification}

One $\mathrm{ml}$ of diluted (1/1000) urine specimen was used for culturing on CLED, Blood, and Mac Conkey agar. Specimen was inoculated using a calibrated loop. The plate was incubated at $35^{\circ} \mathrm{C}$ for $24 \mathrm{hrs}-48 \mathrm{hrs}$.

Specimens with more than $10^{5}$ colony forming unit [CFU]/ $\mathrm{ml}$ were considered as positive samples. The isolates were identified based on colony morphology on culture plates, gram's staining and by standard biochemical tests. Antibiotic susceptibility testing was done by standard methods according to CLSI guidelines.

\section{Antimicrobial susceptibility testing}

Antibiotic susceptibility testing was performed by the Kirbey bauer disc diffusion method on MHA (Mueller hinton agar) according to CLSI protocols. The diameters of zone of inhibition were interpreted according to CLSI standards for each organism. Media and disks were tested for quality control with standard strains. 


\section{Detection of ESBL production in GNB}

\section{Screening method}

Isolates of gram negative bacilli showing the following resistance pattern were considered to be possible ESBL producing strains.

\section{CLSI phenotypic confirmation method}

With a sterile bacterial loop 3 - 5 identical colonies were picked from a fresh overnight grown pure culture and inoculated into $5 \mathrm{ml}$ of nutrient broth. The broth was incubated at $35^{0} \mathrm{C}$ for $2-4 \mathrm{hrs}$ and the turbidity matched with 0.5 Mc Farlands standard. Lawn culture of the test organism was made on to MHA plate [Hi media]. Antibiotic discs Ceftazidime [CAZ 30microgram] and Ceftazidime/ Clavulanic acid [CAZ/CA 30micro gram/10micro gram] [Himedia, Mumbai] were placed onto the plate and incubated at $35^{0} \mathrm{C}$ overnight. $\mathrm{A} \geq 5 \mathrm{~mm}$ increase in zone diameter for Ceftazidime tested in combination with clavulanic acid versus its zone when tested alone confirmed an ESBL producing organisms (Hussam et al., 2009).

\section{Double disk diffusion synergy test}

In this test disks of cefotaxime, ceftazidime and Amoxycillin-clavulanic acid [20 microgram /10 micro gram] were kept 25 $30 \mathrm{~mm}$ apart from center to center on an inoculated MHA.

A clear extension of the edge of inhibition zone of Cephalosporin towards Amoxycillinclavulanic acid disc was interpreted as positive for ESBL production (Chaudhary et al., 2004).

\section{Results and Discussion}

The study undertaken at the Institute of Microbiology, Madras Medical College,
Chennai among samples received from patients of Rajiv Gandhi Government General Hospital, Chennai with cUTI showed the following results.120 Patients were included in this study.

Complicated urinary tract infection is a condition where there are structural and functional abnormalities of the urinary tract. The uropathogens isolated in c UTI varies considerably from that in uncomplicated UTI (Mahesh et al., 2010).

The amount of drug resistance in these isolates is very high. Hence this study was conducted to determine the bacterial profile and the drug resistance pattern in these isolates.

The study was conducted at Institute of Microbiology, Madras Medical College and a total of 120 patients were included in this study.

The maximum number of patients were present in the age group 51yrs -60yrs [25\%], followed by 41yrs - 50yrs group [17\%] [Table 2]. In the study conducted by Mahesh et al., (2010) the maximum patients were seen in 60-69yrs group with $22.5 \%$ followed by $50-59 y r s$ group with $21 \%$. This was in comparison with the present study (Mahesh $e t$ al., 2010).

Males contributed the maximum number of patients with $67.5 \%$ and females constituting $32.5 \%$. The female to male prevalence ratio was 1: 2.1 [Table 1]. This was in comparison with the study conducted by Mahesh et al., where the males were the maximum numbers, with female to male prevalence ratio of 1:1.63 (Mahesh et al., 2010).

Of the total 120 cases of cUTI, Diabetes mellitus [34.17\%] was the most common risk factor contributing to the infection. This was 
followed by renal calculi [15\%], BPH [10\%], post renal transplant [9.17\%], bladder tumour and nephrotic syndrome each constituting $5.83 \%$ and the other cases were HUN, Stricture urethra each constituting $4.17 \%$ and $5 \%$ respectively [Table 5]. This was similar to the study conducted by Mahesh et al., (2010) where the Diabetes mellitus was the most common risk factor with $42.6 \%$. This was in comparison with this study. However the renal calculi were found in only $2 \%$ of the cases.

The majority of the patients presented with dysuria and was found in $37.5 \%$ patients. The next presentation was with fever in $30.8 \%$; followed by pain abdomen, frequency of micturition, hematuria, oliguria in the decreasing order of presentation [Table 4]. These symptoms were comparable with the study by Mahesh et al., (2010)

In this study E. coli [40.83\%] topped the list of organisms causing c UTI [Table 6]. The results from world-wide review are comparable to this study. Peterson et al., (Peterson et al., 2007) in their study done in USA have reported $E$. coli as the most common organism causing cUTI. A review by Lindsay Nicolle et al., (1997) on c UTI reported $E$. coli as the most common uropathogen with a worldwide prevalence rate of $21-54 \%$. The second most common organism is Klebsiella species with $33.33 \%$ followed by Pseudomonas species 7.5\%, Acinetobacter species and Enterococcus faecalis each with $5.84 \%$, Citrobacter freundii 2.5\%, Staphylococcus aureus $3.33 \%$ and finally single isolate of Proteus vulgaris with $0.83 \%$ were isolated in this study.

$65.6 \%$ of E. coli and $62.3 \%$ of Klebsiella spp were ESBL positive [Table 9]. This was in comparison with the study conducted by Mahesh et al., in a tertiary care centre in Bangalore where the ESBL positive E. coli and Klebsiella spp were $66.77 \%$ and $60.27 \%$ respectively (Mahesh et al., 2010).

The E. coli isolated in these patients were 88\% sensitive to Amikacin, $77 \%$ sensitive to Nitrofurantoin, and were $26 \%$ sensitive to Cephalosporin, and $10 \%$ sensitive to Ofloxacin. They were $100 \%$ sensitive to Imipenem [Table 7]. In a similar study conducted by Mahesh et al., (2010) only 20\% E. coli were sensitive to ofloxacin. The resistant rate of the isolates to Amikacin, Gentamicin and Nitrofurantoin were also higher. The high resistance noted is similar to the present study.

\section{Detection of ESBL production in GNB}

\begin{tabular}{|l|l|}
\hline Antibiotics & Zone diameter for possible ESBL Producing strain \\
\hline Ceftazidime 30micro gram & $\leq 22 \mathrm{~mm}$ \\
\hline Cefotaxime 30 micro gram & $\leq 27 \mathrm{~mm}$ \\
\hline
\end{tabular}

Table.1 Gender distribution of patients with c UTI (n-120)

\begin{tabular}{|l|l|l|}
\hline \multicolumn{1}{|c|}{ Gender } & No of patients & Percentage \\
\hline Male & 81 & $67.5 \%$ \\
\hline Female & 39 & $32.5 \%$ \\
\hline Total & 120 & $100 \%$ \\
\hline
\end{tabular}

Males contributed to maximum number of isolates with $67.5 \%$. 
Table.2 Age and sex distribution of patients with c UTI $(n=120)$

\begin{tabular}{|l|l|l|c|c|}
\hline $\begin{array}{c}\text { Age } \\
\text { group }\end{array}$ & $\begin{array}{c}\text { Male[n=81] } \\
\text { No \% }\end{array}$ & $\begin{array}{c}\text { Female [n=39] } \\
\text { No \% }\end{array}$ & Total [n=120] & No. \% \\
\hline $12-20$ & $1(1 \%)$ & $6(15 \%)$ & 7 & $5.5 \%$ \\
\hline $21-30$ & $18(22 \%)$ & $4(10 \%)$ & 22 & $18 \%$ \\
\hline $31-40$ & $7(8.5 \%)$ & $11(28 \%)$ & 18 & $15 \%$ \\
\hline $41-50$ & $13(16 \%)$ & $7(17.5 \%)$ & 20 & $17 \%$ \\
\hline $51-60$ & $22(27 \%)$ & $8(20.5 \%)$ & 30 & $25 \%$ \\
\hline $61-70$ & $12(15 \%)$ & $3(8 \%)$ & 15 & $12.5 \%$ \\
\hline $71-80$ & $6(7 \%)$ & - & 6 & $5 \%$ \\
\hline $81-90$ & $2(2.5 \%)$ & - & 2 & $2 \%$ \\
\hline Total & $81(100 \%)$ & $39(100 \%)$ & 120 & $100 \%$ \\
\hline
\end{tabular}

Maximum number of isolates were obtained in the 51-60 yrs age group.

Table.3 Uropathogens isolated in cUTI $(n=120)$

\begin{tabular}{|l|l|l|}
\hline Microorganisms & No of patients & Percentage \\
\hline GNB & 109 & $90.83 \%$ \\
\hline GPC & 11 & $9.17 \%$ \\
\hline Total & 120 & $100 \%$ \\
\hline
\end{tabular}

The Gram Negative Bacilli constituted $90.83 \%$ of isolates and Gram Positive Cocci constituted $9.17 \%$ of isolates.

Table.4 Symptoms in cUTI patients $(\mathrm{n}=120)$

\begin{tabular}{|l|l|l|}
\hline Symptoms & No of patients & percentage \\
\hline Dysuria & $45(37.5 \%)$ \\
\hline Fever & $37(30.8 \%)$ \\
\hline Pain abdomen & $23(19.16 \%)$ \\
\hline Frequency of micturition & $15(12.5 \%)$ \\
\hline Hematuria & $6(5 \%)$ \\
\hline Oliguria & $3(2.5 \%)$ \\
\hline
\end{tabular}

Dysuria and fever were the predominant symptoms in these patients. 
Table.5 Distribution of diseases associated with c UTI patients [ $\mathrm{n}=120]$

\begin{tabular}{|l|c|l|}
\hline \multicolumn{1}{|c|}{ Name of the Disease } & No of patients & \multicolumn{1}{c|}{ Percentage } \\
\hline Diabetes mellitus & 41 & $34.17 \%$ \\
\hline Renal Calculi & 12 & $10 \%$ \\
\hline BPH & 12 & $10 \%$ \\
\hline Bladder tumour & 7 & $5.83 \%$ \\
\hline Nephrotic syndrome & 7 & $5.83 \%$ \\
\hline HUN & 5 & $4.17 \%$ \\
\hline Stricture urethra & 6 & $5 \%$ \\
\hline Vesical calculi & 3 & $2.5 \%$ \\
\hline Ureteric calculi & 3 & $2.5 \%$ \\
\hline Nephritic syndrome & 2 & $1.67 \%$ \\
\hline PKD & 2 & $1.67 \%$ \\
\hline Phimosis & 2 & $1.67 \%$ \\
\hline Post Renal Transplant & 11 & $9.17 \%$ \\
\hline Carcinoma penis & 1 & $0.83 \%$ \\
\hline Testicular epididymo orchitis & 1 & $0.83 \%$ \\
\hline Renal abscess & 1 & $0.83 \%$ \\
\hline ARF & 1 & $0.83 \%$ \\
\hline Meatal stenosis & 1 & $0.83 \%$ \\
\hline Perinephric abscess & 1 & $0.83 \%$ \\
\hline Carcinoma prostate & 1 & $0.83 \%$ \\
\hline Acute prostatitis & 1 & $0.83 \%$ \\
\hline Cushing's syndrome & 1 & $0.83 \%$ \\
\hline
\end{tabular}

Diabetes mellitus was the commonest associated illness with $34.17 \%$ followed by Renal Calculi with $15 \%$ of the patients (including vesical and ureteric calculi).

Table.6 Uropathogens isolated in cUTI

\begin{tabular}{|l|l|c|}
\hline \multicolumn{1}{|c|}{ Organisms } & No of patients & Percentage \\
\hline E.coli & 49 & $40.83 \%$ \\
\hline K.pneumoniae \& K. Oxytoca & 40 & $33.33 \%$ \\
\hline Pseudomonas aeruginosa & 9 & $7.5 \%$ \\
\hline Acinetobacter spp & 7 & $5.84 \%$ \\
\hline Enterococcus faecalis & 7 & $5.84 \%$ \\
\hline Staph. aureus & 4 & $3.33 \%$ \\
\hline Citrobacter freundii & 3 & $2.50 \%$ \\
\hline Proteus vulgaris & 1 & $0.83 \%$ \\
\hline Total & 120 & $100 \%$ \\
\hline
\end{tabular}

E.coli was the commonest isolate with $40.83 \%$ and Klebsiella spp with $33.33 \%$. The other isolates were Pseudomonas spp, Acinetobacter spp, S. aureus, citrobacter spp \&Proteus vulgaris. 
Table.7 Antimicrobial sensitivity pattern in gram negative bacilli [n=109]

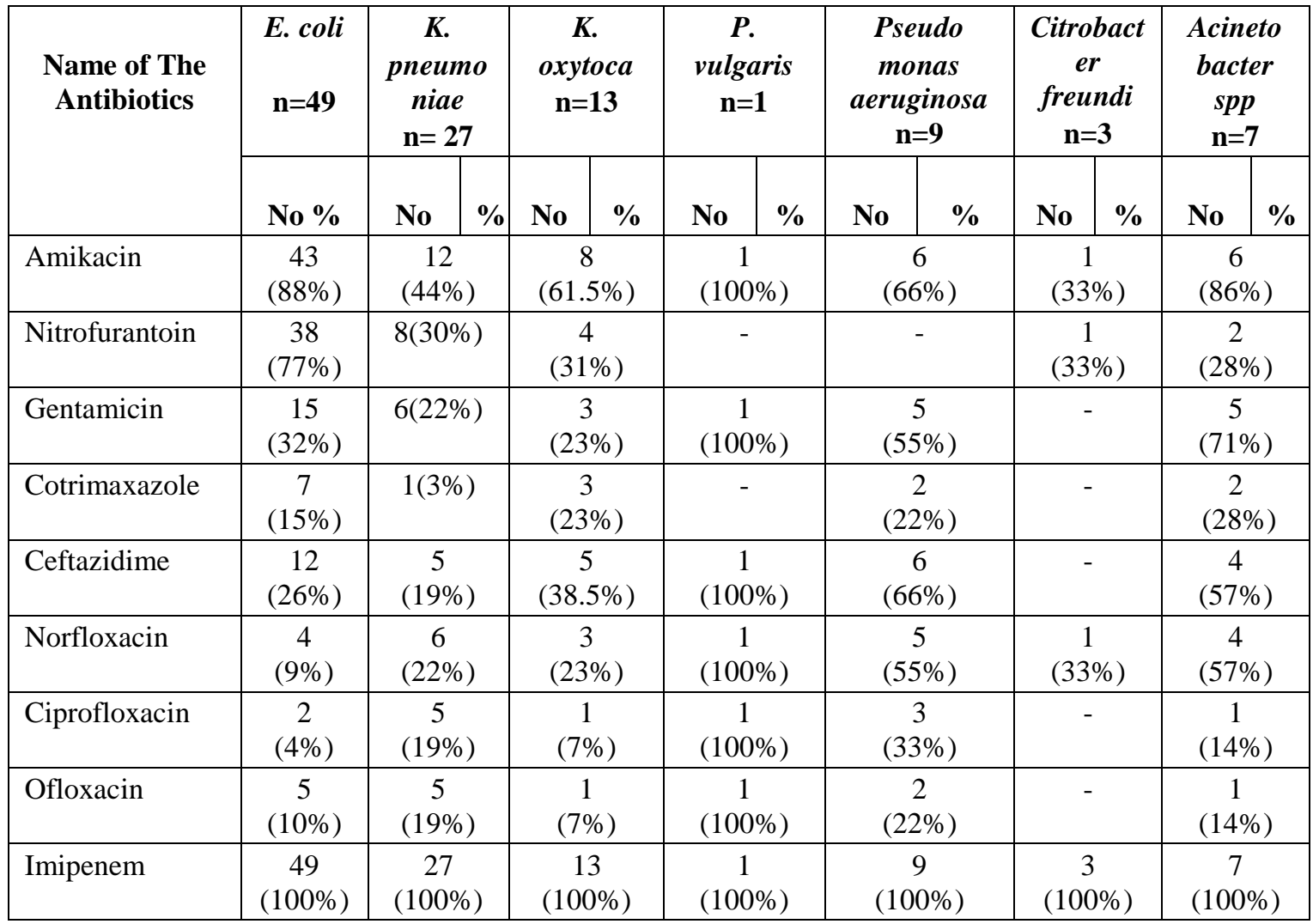

In GNB isolates, Amikacin, Nitrofurantoin and Imipenem had higher sensitivity.

Table.8 Anti-microbial sensitivity pattern in gram positive cocci $[\mathrm{n}=11]$

\begin{tabular}{|c|c|c|c|c|}
\hline \multirow[t]{2}{*}{ Antibiotics } & \multicolumn{2}{|c|}{$\begin{array}{l}\text { Enterococcus } \\
\text { faecalis } \mathrm{n}=7\end{array}$} & \multicolumn{2}{|c|}{ Staph. aureus $\mathrm{n}=4$} \\
\hline & No & $\%$ & No & $\%$ \\
\hline Amikacin & 1 & $14 \%$ & 3 & $75 \%$ \\
\hline Nitrofurantoin & 4 & $57 \%$ & - & - \\
\hline Cotrimoxazole & - & - & 1 & $25 \%$ \\
\hline Ciprofloxacin & 1 & $14 \%$ & 1 & $25 \%$ \\
\hline Erythromycin & 1 & $14 \%$ & 1 & $25 \%$ \\
\hline Amoxycillin & 2 & $26 \%$ & 1 & $25 \%$ \\
\hline $\begin{array}{l}\text { Amoxycillin Clavulanic } \\
\text { Acid }\end{array}$ & 4 & $57 \%$ & 2 & $50 \%$ \\
\hline Cephalexin & 3 & $42 \%$ & 2 & $50 \%$ \\
\hline Oxacillin & & & 1 & $25 \%$ \\
\hline
\end{tabular}

The Gram positive cocci isolated were Enterococci spp and S. aureus which were 7 and 4 isolates respectively. 
Table.9 List of ESBL producing organisms [ $\mathrm{n}=67]$

\begin{tabular}{|l|l|l|l|l|l|}
\hline \multicolumn{1}{|c|}{ Organism } & \multicolumn{1}{c|}{$\begin{array}{c}\text { Screening test } \\
\text { with Cefotaxime }\end{array}$} & \multicolumn{2}{|c|}{$\begin{array}{c}\text { PCDDT } \\
\text { No \% }\end{array}$} & \multicolumn{2}{c|}{$\begin{array}{c}\text { DDST } \\
\text { N0 \% }\end{array}$} \\
\hline E.coli $\mathrm{n}=49$ & 37 & 32 & $65.6 \%$ & 32 & $65.6 \%$ \\
\hline Klebsiella spp $\mathrm{n}=40$ & 30 & 25 & $62.5 \%$ & 25 & $62.5 \%$ \\
\hline
\end{tabular}

The ESBL production in E.coli were higher than Klebsiella spp.

The Klebsiella pnemoniae isolated in these patients were $44 \%$ sensitive to Amikacin, $30 \%$ sensitive to Nitrofurantoin and19\% sensitive to cephalosporin and Fluoroquinolones [Table 7].

The Klebsiella oxytoca isolated in these patients were $61.5 \%$ sensitive to Amikacin, $31 \%$ sensitive to Nitrofurantoin,38.5\% sensitive to Cephalosporin and $7 \%$ sensitive to Fluoroquinolones [Table 7]. Both the Klebsiella spp were $100 \%$ sensitive to Imipenem. In a similar study conducted by Mahesh et al., the sensitive rate to Fluoroquinolones among Klebsiella spp was $40 \%$.

The resistance -rate was higher for Amikacin, Garamycin, Nitrofurantoin in these isolates (Mahesh et al., 2010). The high resistance noted is similar to the present study. The Pseudomonas aeruginosa was the third commonest isolate in these patients. They were $66 \%$ sensitive to Amikacin and Cephalosporin, $33 \%$ sensitive to Ciprofloxacin and $22 \%$ sensitive to Ofloxacin [Table 7]. In a similar study by Mahesh et al., $74.5 \%$ resistance rate to Fluoroquinolones and $64.7 \%$ resistance to Amikacin and Gentamicin were noted for Pseudomonas spp. This was in comparison to the present study.

In the present study, Pseudomonas spp were $100 \%$ sensitive to Imipenem whereas $70 \%$ of the isolates were sensitive to Carbapenems in the study by Mahesh et al., This is not coherent with the present study. The Acinetobacter spp isolated in this study were
86\% sensitive to Amikacin, $57 \%$ sensitive to Cephalosporin and Norfloxacin; 14\% sensitive to Fluoroquinolones and 28\% sensitive to Nitrofurantoin and Cotrimoxazole and 100\% sensitive to Imipenem [Table 7].

The Citrobacter freundii constituted 3 isolates in this study. They were $33 \%$ sensitive to Amikacin, Nitrofurantoin and Norfloxacin. They were $100 \%$ sensitive to Imepenem [Table 7].

The Proteus vulgaris was the single isolate in this study. It was $100 \%$ sensitive to Amikacin, Gentamicin, Cephalosporins, Fluoroquinolones and Imipenem [Table 7].

The Enterococcus faecalis constituted 7 isolates in this study. They were 14\% sensitive to Amikacin, and Ciprofloxacin. They were $57 \%$ sensitive to Nitrofurantoin and $42 \%$ sensitive to Cephalexin. They were $100 \%$ resistant to Cotrimoxazole [Table 8]. In a similar study by Mahesh et al., 28.5\% resistance to Fluoroquinolones and 85\% resistant to Cotrimoxazole was noted. This was in comparison to the present study (Mahesh et al., 2010).

The $S$. aureus constituted 4 isolates in this study. They were $75 \%$ sensitive to Amikacin. They were 50\% sensitive to Amoxicillin Clavulanic acid and cephalexin.3 isolates were oxacillin resistant. The isolates were 100\% sensitive to Carbapenems. The highly sensitive rate to Carbapenems, could be due to it usage pattern in our institution. It is an expensive drug, used in critically ill inpatients 
where the administration of approximate doses for the full duration is ensured. The high rate of resistance in Cephalosporins and Fluoroquinolones are multifactorial including lack of protocol for c UTI and low compliance of patients in completing the antibiotic course. The increasing rate of multidrug resistant uropathogens and ESBL producing organism pose a serious threat in treating cUTI.

The removal of the stasis factor in these patients with complicated UTI will contribute much towards treating these patients. An effective Antibiotic Policy incorporating these results will help in Treatment and improved Prognosis of these patients.

\section{References}

Bailey, and Scott's Diagnostic Microbiology 12th edition chapter -57 , page 845-850.

Chaudbary, U., Agarwal R ESBL An emerging threat to clinical therapeutics IJMM 2004; 22 (2) $75-80$.

Foxman, B. Epidemiology of urinary tract infections; incidence, morbidity, and economic costs. Am J Med 2002; 113 (suppl IA0 5S- 13S [M-90].

Hussam S. Khanfur, Khalid M. Bindayana ESBL in E.coli; tends in the hospital and community settings Journal of Infectious diseases 2009; 3(4): 295-299.

Konemans colour atlas and text book of diagnostic Microbiology page 82-85.

Mackie, and Mc Cartney, practical Medical Microbiology Cha 5, 6, 7pg95-150.

Mahesh. E et al., Ramesh.D, Indumathi V.A, Punith K, Kirthi Raj and Anupama H.A, Complicated urinary tract infection in a tertiary care center in South India. Al Ameen J Med Sci, Volume 3, No. 2, 2010 [J-1].

National Committee for clinical, Laboratories standards, disk diffusion, supplemental tables M 100- S 13. NGCLS, Wayen, PA, USA, 2003 [ESBL -1].

Nicolle, L.E., A practical guide to the managemet of complicated urinary tract infections. Drugs 1997; 53:583-92. [J-1].

Performance standards for antimicrobial susceptibility testing; 15th informational supplement [M 100- SIS] 2005 National GCLS WAYNE PA, USA, NC for CLS] [ESBL -2].

Peterson, J., Kaul S, Khashhab M, Fisher A, Kahn JB. Identification and pretherapy susceptibility of pathogens in patients with complicated urinary tract infection or acute pyelonephritis enrolled in a clinical study in the United State from Nov 2004-April 2006.Clin.ther 2007 Oct 29(10): 2215-21.[J-1].

Wang, J.M., Lim HK, Pang KK. Emphysematous pyelonephritis. Scand J Urol Nephrol. 2007; 41:223-229 [m-158].

Wheat, L.J. Infections and Diabetes mellitus. Diabetes Care 1980; 3; 187. [S-87].

Wu, H.C., Chang CH, Lai MM, et al, Using Tc99m DMSA renal cortex scan to detect renal damage in women with type 2 diabetes. J Diabetes Complications 2003; 17:297[s-94].

Zhou, G., Mo WJ, Sebbel P, et al., Uroplakin Ia is the urothelial receptor for uropathogenic Escherichia coli ; evidence from in vitro Fim $\mathrm{H}$ binding. J Cell SCI, 2001;114;4095-4103 [M-31].

Zorc, J.J., Kiddoo DA, Shaw KN. Diagnosis and management of pediatric urinary tract infections. Clin Micro Rev. 2005; 18: 417422. [M-83].

\section{How to cite this article:}

Natesan, B. and Manjula, R. 2017. A Study on Bacterial Profile and Antibiotic Susceptibility Pattern in Complicated Urinary Tract Infection in a Tertiary Care Centre. Int.J.Curr.Microbiol.App.Sci. 6(10): 2286-2294. doi: https://doi.org/10.20546/ijcmas.2017.610.271 